\title{
Arthritis Associated with Inflammatory Bowel Disease
}

\section{Sacroiliitis}

- A 39-year-old patient

- Right-sided back pain

- Patient affected by Crohn's disease
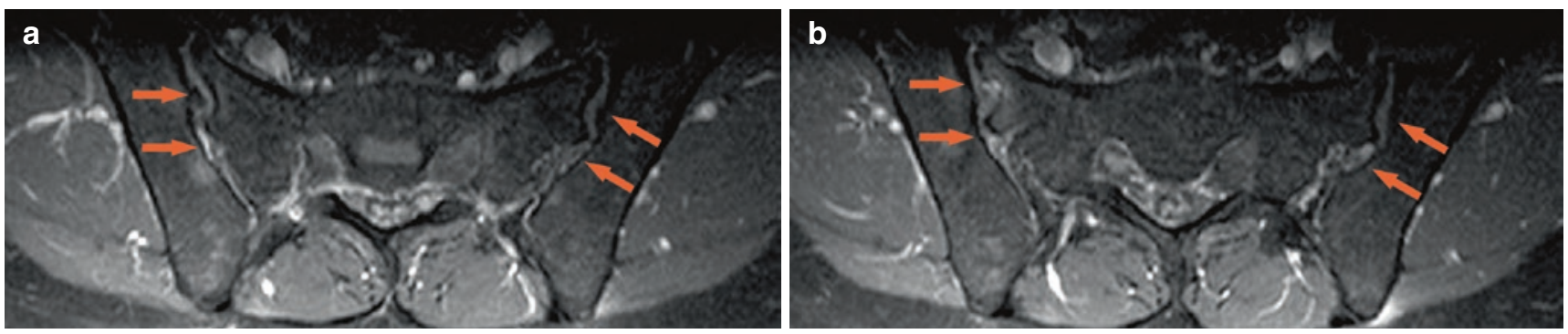

Fig. 1 Axial SE T1-weighted images with fat saturation following the administration of contrast medium (a-b). Right sacroiliitis. Note contrast enhancement in the joint space, indicating an active inflammatory stage of the process. Note also bone erosion on the sacral side 